\title{
BAYESIAN ESTIMATION FOR TWO WEIBULL POPULATIONS BASED ON JOINT RECORDS
}

\section{TINGTING HU}

School of Information and Science Technology

Jinan University

Guangzhou 510632

P. R. China

e-mail: 1825638419@qq.com

\begin{abstract}
In this paper, we discussed the Bayesian estimation for two Weibull populations with same shape parameter based on observed lower-joint records, inter-record times and record indicators. As using a general joint prior will lead to computational complexity, we use Soland's method to simplify the analysis. Finally, a simulation study is conducted to evaluate the Bayesian method.
\end{abstract}

\section{Introduction}

The record values were first introduced by Chandler [1]. Since then, there have been a lot of literature on statistical inference based on record values, such as Shorrock [2], Arashi and Emadi [3], Afshari [4], Kizılaslan and Nadar [5], etc. 
Let $\left\{X_{i}, i \geq 1\right\}$ be a sequence of independently and identically distributed random variables from a same population. The first record and record time are set as $L_{1}=X_{1}$ and $T_{1}=1$, respectively. We call $\left\{L_{j}, j \geq 1\right\}$ lower records if $L_{j}=X_{T_{j}}$, where $T_{j}=\min \left\{i: X_{i}<L_{j-1}\right\}$, $j \geq 2$. The inter-record times are then defined as $K_{j}=T_{j+1}-T_{j}$. An analogous definition can be provided for upper record values.

For the record values from one population, there have been many related research literature. As the arising of record values is relatively rare, considering the records from multiple sequences would be effective because it results in more record data. Also, in lots of practical applications, based on the number and values of records, comparing two populations will be meaningful. Hence, some scholars have begun to study joint records. Belaghi et al. [6] proposed joint records first, and discussed exact nonparametric inference based on joint records under the assumption that the random variables have common underlying distribution. Volterman et al. [7] studied the statistical inference problem of parameter for two exponential distributions based on lower-joint records.

The definition of joint records from two independent sequences is as follows: Let $\left\{\left(X_{i}, Y_{i}\right), i \geq 1\right\}$ be two independent sequences of independent and identically distributed (IID) continuous random variables. We call the sequence $\left\{W_{i}, i \geq 1\right\}$ the lower-joint records, which is the sequence of lower records from the sequence $\left\{\min \left(X_{i}, Y_{i}\right), i \geq 1\right\}$. Again, let $T_{i}$ be the record times and $K_{i}$ represent the inter-record times as before. Also, we define indicators $\left\{Z_{i}, i \geq 1\right\}$ with $z_{i}=1$ or 0 according as $w_{i}$ is from sequence $X$ or sequence $Y$.

The discussion of joint records in [7] was supposed that the records have a base distribution-exponential distribution. It is natural to think of whether it can be extended to more complex models. In this paper, we 
will assume that the $X$ and $Y$ sequences are from two Weibull distribution. Note that the inference of the parameters are based on the observed first $r$ lower-joint records, the indicators and inter-record times.

\section{Main Results}

\subsection{Weibull model}

Let $\left\{X_{i}, i \geq 1\right\}$ and $\left\{Y_{i}, i \geq 1\right\}$ be two independent continuous random sequences from two different populations with density and distribution functions as

$$
F_{j}(x)=1-e^{-\left(\frac{x}{\alpha_{j}}\right)^{\beta}} \text { and } f_{j}(x)=\frac{\beta}{\alpha_{j}}\left(\frac{x}{\alpha_{j}}\right)^{\beta-1} e^{-\left(\frac{x}{\alpha_{j}}\right)^{\beta}},
$$

where $x>0, \beta>0, \alpha_{j}>0$ for $j=1,2 . \alpha_{j}$ is scale parameter, $\beta$ is shape parameter. Suppose we have observed the first $r$ lower-joint records $(w)$, record indicators $(z)$, and the first $r-1$ inter-record times (k) from the two sequences $X$ and $Y$. For brevity, the last inter-record times $k_{r}$ is fixed to 1 . Then following Volterman et al. [7], the likelihood function of the data set $(W, Z, K)$ is

$$
f(w, z, k)=\prod_{i=1}^{r}\left[\bar{F}_{1}\left(w_{i}\right) \bar{F}_{2}\left(w_{i}\right)\right]^{k_{i}-1}\left[f_{1}\left(w_{i}\right) \bar{F}_{2}\left(w_{i}\right)\right]^{z_{i}}\left[f_{2}\left(w_{i}\right) \bar{F}_{1}\left(w_{i}\right)\right]^{1-z_{i}},
$$

where $w_{1}>w_{2}>\cdots>w_{r}>0, z \in\{0,1\}^{r}, k \in \mathbb{N}^{r-1}$, and $\bar{F}=1-F$ is survival functions. Substitute (1) into (2), then we get the likelihood function of data set $(W, Z, K)$ as:

$$
\begin{gathered}
f\left(\alpha_{1}, \alpha_{2}, \beta ; w, z, k\right)=\left(\frac{\beta}{\alpha_{1}^{\beta}}\right)^{m_{r}}\left(\frac{\beta}{\alpha_{2}^{\beta}}\right)^{r-m_{r}} \prod_{i=1}^{r} w_{i}^{\beta-1} \exp \left\{-\sum_{i=1}^{r} k_{i}\left[\left(\frac{w_{i}}{\alpha_{1}}\right)^{\beta}+\left(\frac{w_{i}}{\alpha_{2}}\right)^{\beta}\right]\right\} \\
w_{1}>w_{2}>\cdots>w_{r}>0, \quad z \in\{0,1\}^{r}, \quad k \in \mathbb{N}^{r-1}
\end{gathered}
$$

where $m_{r}=\sum_{i=1}^{r} z_{i}$. The last inter-record time $k_{r}=1$. 
Theorem 1. We get the following results:

(i) $Z$ is independent of $(W, K)$;

(ii) $Z_{1}, Z_{2}, \ldots, Z_{r} \stackrel{\text { iid }}{\sim}$ Bernoulli $\left(\alpha_{2}^{\beta} /\left(\alpha_{1}^{\beta}+\alpha_{2}^{\beta}\right)\right)$;

(iii) $\sum_{i=1}^{r} Z_{i} \sim$ Binomial $\left(r, \alpha_{2}^{\beta} /\left(\alpha_{1}^{\beta}+\alpha_{2}^{\beta}\right)\right)$;

(iv) The joint density of $(W, K)$ is

$f(w, k)=\left(\frac{\beta}{\alpha_{1}^{\beta}}+\frac{\beta}{\alpha_{2}^{\beta}}\right)^{r} \prod_{i=1}^{r} w_{i}^{\beta-1} \exp \left\{-\sum_{i=1}^{r} k_{i} w^{\beta}{ }_{i}\left(\frac{1}{\alpha_{1}^{\beta}}+\frac{1}{\alpha_{2}^{\beta}}\right)\right\}$.

\subsection{Bayesian estimation}

In this subsection, we consider Bayesian estimation for the parameters of the Weibull distribution based on joint records. Specifying a general joint prior for $\alpha$ and $\beta$ may lead to computational complexities. In order to simplify the analysis, we used Soland's method [8]. For more information about Soland's method, one can also refer to Doostparast [9].

We re-parameterize the model first, as $\theta_{1}=1 / \alpha_{1}^{\beta}$ and $\theta_{2}=1 / \alpha_{2}^{\beta}$. Next, we will consider the Bayes estimators of $\alpha_{1}$ and $\alpha_{2}$ based on this new parameterization. In this case our likelihood function of $(W, Z, K)$ can be expressed as

$$
f\left(\theta_{1}, \theta_{2}, \beta ; w, z, k\right) \propto \theta_{1}^{m_{r}} \theta_{2}^{r-m_{r}} \beta^{r} \prod_{i=1}^{r} w_{i}^{\beta-1} \exp \left\{-\sum_{i=1}^{r} k_{i} w_{i}^{\beta}\left(\theta_{1}+\theta_{2}\right)\right\} .
$$

In Soland's method, we will assume a discrete prior for $\beta$, denoted by $\beta_{1}, \beta_{2}, \ldots, \beta_{s} \quad\left(s\right.$ is finite) with prior probability $\xi_{1}, \xi_{2}, \ldots, \xi_{s}$, respectively, and meets

$$
\operatorname{Pr}\left(\beta=\beta_{j}\right)=\xi_{j}, j=1,2, \ldots, s, \xi_{j} \in[0,1] \text { and } \sum_{j=1}^{s} \xi_{j}=1 .
$$


Also, suppose $\theta_{1}$ and $\theta_{2}$ given that $\beta=\beta_{j}$ are independent and have

$$
\pi\left(\theta_{i} \mid \beta=\beta_{j}\right)=\frac{\left(b_{i} \beta_{j}\right)^{a_{i}}}{\Gamma\left(a_{i}\right)}\left(\theta_{i}\right)^{a_{i}-1} e^{-\theta_{i}\left(b_{i} \beta_{j}\right)}, \theta_{i}>0, i=1,2, j=1,2, \ldots, s,
$$

where $\left(a_{i}, b_{i}\right)$ are positive hyperparameters.

Then we can obtain the conditional posterior pdf of $\theta$ given that $\beta=\beta_{j}$ and $(w, z, k)$ is

$$
\begin{aligned}
\pi\left(\theta_{i} \mid \beta=\beta_{j}, w, z, k\right) & =\frac{f\left(\theta_{1}, \theta_{2}, \beta ; w, z, k\right) \pi\left(\theta_{1} \mid \beta=\beta_{j}\right) \operatorname{Pr}\left(\beta=\beta_{j}\right)}{\int_{0}^{\infty} f\left(\theta_{1}, \theta_{2}, \beta ; w, z, k\right) \pi\left(\theta_{1} \mid \beta=\beta_{j}\right) \operatorname{Pr}\left(\beta=\beta_{j}\right) d \theta_{i}} \\
& =\frac{\left(B_{i j}\right)^{A_{i}}}{\Gamma\left(A_{i}\right)}\left(\theta_{i}\right)^{A-1} e^{-\theta_{i} B_{i j}}, \theta_{i}>0, i=1,2
\end{aligned}
$$

From above formula (7), we have $\left(\theta_{i} \mid \beta=\beta_{j}, w, z, k\right) \sim \Gamma\left(A_{i}, B_{i j}\right)$, where

$$
A_{1}=m_{r}+a_{1}, \quad A_{2}=a_{2}+r-m_{r}, \quad B_{1 j}=b_{1} \beta_{j}+\sum_{i=1}^{r} k_{i} w_{i}{ }^{\beta_{j}},
$$

and

$$
B_{2 j}=b_{2} \beta_{j}+\sum_{i=1}^{r} k_{i} w_{i}^{\beta_{j}}
$$

And, the marginal posterior pdf of $\beta=\beta_{j}$ is obtained as

$$
\begin{aligned}
\pi_{j} & =\operatorname{Pr}\left(\beta=\beta_{j} \mid w, z, k\right) \\
& =C \int_{0}^{\infty} \int_{0}^{\infty} f\left(\theta_{1}, \theta_{2}, \beta ; w, z, k\right) \pi\left(\theta_{1} \mid \beta=\beta_{j}\right) \pi\left(\theta_{2} \mid \beta=\beta_{j}\right) \operatorname{Pr}\left(\beta=\beta_{j}\right) d \theta_{1} d \theta_{2} \\
& =C \frac{\Gamma\left(A_{1}\right) \Gamma\left(A_{2}\right) \xi_{j} b_{1}{ }^{a_{1}} b_{2}{ }^{a_{2}} \beta_{j}^{a_{1}+a_{2}+r}}{\left(B_{1 j}\right)^{A_{1}}\left(B_{2 j}\right)^{A_{2}}} \prod_{i=1}^{r} w_{i}^{\beta_{j}-1}
\end{aligned}
$$




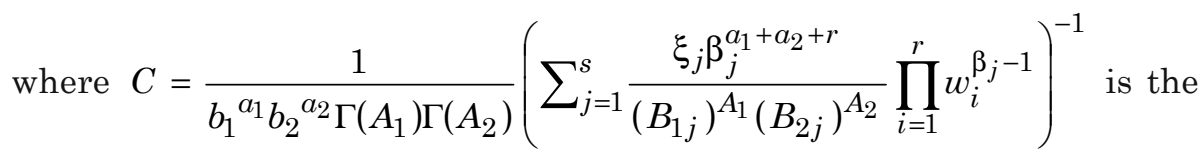
normalizing constant. Thus, under the SE loss functions, we can get the Bayes estimators of $\theta_{1}, \theta_{2}$, and $\beta$ as

$$
\begin{aligned}
\hat{\theta}_{1(B S)} & =\int_{0}^{\infty} \theta_{1} \int_{0}^{\infty} \sum_{j=1}^{s}\left(\prod_{i=1}^{2} \pi\left(\theta_{i} \mid \beta=\beta_{j}, w, z, k\right) \operatorname{Pr}\left(\beta=\beta_{j} \mid w, z, k\right) d \theta_{2} d \theta_{1}\right. \\
& =A_{1} \sum_{j=1}^{s} \pi_{j} / B_{1 j} ; \\
\hat{\theta}_{2(B S)}= & \int_{0}^{\infty} \theta_{2} \int_{0}^{\infty} \sum_{j=1}^{s}\left(\prod_{i=1}^{2} \pi\left(\theta_{i} \mid \beta=\beta_{j}, w, z, k\right) \operatorname{Pr}\left(\beta=\beta_{j} \mid w, z, k\right) d \theta_{1} d \theta_{2}\right. \\
= & A_{2} \sum_{j=1}^{s} \pi_{j} / B_{2 j} ; \quad \hat{\beta}_{(B S)}=\sum_{j=1}^{s} \beta_{j} \pi_{j} .
\end{aligned}
$$

The Bayes estimators of $\alpha_{1}$ and $\alpha_{2}$ are then available as

$$
\hat{\alpha}_{1(B S)}=\hat{\theta}_{1(B S)}^{-\frac{1}{\hat{\beta}_{(B S)}}} \text { and } \hat{\alpha}_{2(B S)}=\theta_{2(B S)}^{-\frac{1}{\hat{\beta}_{(B S)}}}
$$

To use the priors distribution on $\theta_{1}, \theta_{2}$ and $\beta$ discussed before, we need to determine the hyperparameters of $(a, b)$ and $\left(\beta_{j}, \xi_{j}\right), j=1,2, \ldots, s$ first. The determination of $\left(\beta_{j}, \xi_{j}\right)$ is quite straightforward. As for the value of $(a, b)$, we use the method suggested in [9] to determine. Assuming that based on prior beliefs, the conditional expectation as well 
as the variance of $\theta$ given a special value of $\beta\left(\right.$ as, $\left.\beta=\beta_{h}\right)$ are known. So, the following equations are available:

$$
\mu_{h}=E\left(\theta \mid \beta=\beta_{h}\right)=a /\left(b \beta_{h}\right), \sigma_{h}^{2}=\operatorname{Var}\left(\theta \mid \beta=\beta_{h}\right)=a /\left(b^{2} \beta_{h}^{2}\right)=\mu_{h} /\left(b \beta_{h}\right) .
$$

By solving above Equations (12), we get

$$
a=\mu_{h}^{2} / \sigma_{h}^{2}, \quad b=\mu_{h} /\left(\beta_{h} \sigma_{h}^{2}\right) .
$$

From Equations (12) and (13) we can know, a larger value for $b$ represents more prior information of the parameter $\alpha$ when $\mu_{h}$ fixed.

\section{Numerical Computations}

In this section, the performance of Bayes estimators $\hat{\phi}$ under the SE loss functions are evaluated numerically in terms of the estimated risks (ERs) for different numbers $\{r=3(1) 10\}$ of lower-joint records. In the simulation, by using the Monte Carlo method, the Bayes estimator $\hat{\phi}$ of the desired parameter $\phi$ is computed and the estimated risks (ERs) defined as

$$
\operatorname{ER}(\hat{\phi})=\sum_{i=1}^{N 1}\left(\hat{\phi}_{i}-\phi\right)^{2} / N 1
$$

Algorithm implementation

(1) Supposed that $\beta_{h}=1,\left(\mu_{1 h}, \sigma_{1 h}^{2}, \mu_{2 h}, \sigma_{2 h}^{2}\right)=(1,0.1,1,0.05)$, then we can obtain the values of $\left(a_{1}, b_{1}\right)$ and $\left(a_{2}, b_{2}\right)$ by Equations (13).

(2) Assuming $\beta$ falls in an interval $(0,3)$. We generate $\beta_{i}$ from discrete uniform distribution on $\{0.5,1,1.5,2,2.5,3\}$. And from the priors distribution $\Gamma\left(a_{1}, b_{1} \beta_{i}\right)$ and $\Gamma\left(a_{2}, b_{2} \beta_{i}\right)$, we generate $\theta_{1 i}$ and $\theta_{2 i}$, respectively. And then, we can obtain $\alpha_{1 i}$ and $\alpha_{2 i}$ according to Equations (12). 
(3) Two random samples both of sizes $m$ are generated from Weibull $\left(\alpha_{1 i}, \beta_{i}\right)$ and Weibull $\left(\alpha_{2 i}, \beta_{i}\right)$ for $X$ and $Y$, respectively. Then, we obtain the lower-joint records sample based on the two random samples $X$ and $Y$.

(4) Based on the simulated lower-joint records sample obtained in Step (3). We obtained Bayes estimators $\phi_{i}$ under the SE loss functions.

(5) Repeated Steps (1)-(4) $t=10000$ times and ERs are calculate using Equation (14).

Table 1 tabulates the ERs of estimators $\hat{\alpha}_{1}, \hat{\alpha}_{2}$, and $\hat{\beta}$. From Table 1 , we know that if sufficient prior information is available, the Bayes estimation method discussed in this paper performs well. When the observed number $(r)$ of joint records increases, the ERs of estimators $\hat{\alpha}_{1}, \hat{\alpha}_{2}$, and $\hat{\beta}$ reduced as expected. In the simulation, we also found that the computational complexity of the Soland's method is greatly reduced. Moreover, for small $r(r=3,4,5)$, the Soland's method is also applicable and effective.

Table 1. The values of ER for the estimates of $\hat{\alpha}_{1}, \hat{\alpha}_{2}$, and $\hat{\beta}$

\begin{tabular}{cccc}
\hline$r$ & $\hat{\alpha}_{1}$ & $\hat{\alpha}_{2}$ & $\hat{\beta}$ \\
\hline 3 & 0.4162 & 0.4247 & 0.1757 \\
4 & 0.3602 & 0.3885 & 0.1596 \\
5 & 0.3117 & 0.3541 & 0.1448 \\
6 & 0.2768 & 0.3279 & 0.1342 \\
7 & 0.2543 & 0.3114 & 0.1270 \\
8 & 0.2442 & 0.3027 & 0.1227 \\
9 & 0.2402 & 0.2982 & 0.1206 \\
10 & 0.2393 & 0.2928 & 0.1179 \\
\hline
\end{tabular}




\section{Illustrate Example}

The data set of air conditioning system failure interval (hours) of a fleet of 13 Boeing 720 jet airplanes proposed by Proschan [10] is applied to the method mentioned in previous chapter. Here, we consider the failure time data sets of planes "7913" and "7914". The corresponding failure time of these two aircraft is as follows:

$X=[97,51,11,4,141,18,142,68,77,80,1,16,106,206,82,54,31,216$, $46,111,39,63,18,191]$;

$Y=[50,44,102,72,22,39,3,15,197,188,79,88,46,5,5,36,22,139$, $210,97,30,23,13,14]$.

The lower-joint records extracted from above airplane data are tabulated in Table 2. The maximum likelihood estimators and Bayes estimators for parameter $\alpha_{1}, \alpha_{2}$, and $\beta$ are given in Table 3 .

Table 2. Joint records extracted from airplane data

\begin{tabular}{cccc}
\hline$j$ & $k_{j}$ & $w_{j}$ & $z_{j}$ \\
\hline 1 & 1 & 50 & 0 \\
2 & 1 & 44 & 0 \\
3 & 1 & 11 & 1 \\
4 & 3 & 4 & 1 \\
5 & 4 & 3 & 0 \\
6 & 1 & 1 & 1 \\
\hline
\end{tabular}

Table 3. MLEs and Bayes estimators for parameters $\alpha_{1}, \alpha_{2}$, and $\beta$

\begin{tabular}{cccc}
\hline Method & $\alpha_{1}$ & $\alpha_{2}$ & $\beta$ \\
\hline MLEs & 42.5575 & 42.5575 & 0.9083 \\
Bayes & 42.7952 & 42.7952 & 0.9744 \\
\hline
\end{tabular}




\section{References}

[1] K. N. Chandler, The distribution and frequency of record values, Journal of the Royal Statistical Society: Series B (Methodological) 14(2) (1952), 220-228.

DOI: https://doi.org/10.1111/j.2517-6161.1952.tb00115.x

[2] R. W. Shorrock, On record values and record times, Journal of Applied Probability 9(2) (1972), 316-326.

DOI: https://doi.org/10.2307/3212801

[3] M. Arashi and M. Emadi, Evidential inference based on record data and inter-record times, Statistical Papers 49(2) (2008), 291-301.

DOI: https://doi.org/10.1007/s00362-006-0013-8

[4] M. Afshari, Bayesian estimation distribution and survival function of records and inter-record times and numerical computation for Weibull model, Thai Journal of Mathematics 9(1) (2011), 75-81.

[5] F. Kizılaslan and M. Nadar, Estimation and prediction of the Kumaraswamy distribution based on record values and inter-record times, Journal of Statistical Computation and Simulation 86(12) (2016), 2471-2493.

DOI: https://doi.org/10.1080/00949655.2015.1119832

[6] R. A. Belaghi, M. N. Asl, H. Bevrani, W. Volterman and N. Balakrishnan, On the distribution-free confidence intervals and universal bounds for quantiles based on joint records, Communications in Statistics - Theory and Methods 47(12) (2018), 2969-2978.

DOI: https://doi.org/10.1080/03610926.2017.1343845

[7] W. Volterman, R. A. Belaghi and N. Balakrishnan, Joint records from two exponential populations and associated inference, Computational Statistics 33(1) (2018), 549-562.

DOI: https://doi.org/10.1007/s00180-017-0761-z

[8] R. M. Soland, Bayesian analysis of the weibull process with unknown scale and shape parameters, IEEE Transactions on Reliability R-18(4) (1969), 181-184.

DOI: https://doi.org/10.1109/TR.1969.5216348

[9] M. Doostparast, M. V. Ahmadi and J. Ahmadi, Bayes estimation based on joint progressive type II censored data under LINEX loss function, Communications in Statistics - Simulation and Computation 42(8) (2013), 1865-1886.

DOI: https://doi.org/10.1080/03610918.2012.683921

[10] F. Proschan, Theoretical explanation of observed decreasing failure rate, Technometrics 5(3) (1963), 375-383.

DOI: https://doi.org/10.2307/1266340 